\title{
Article \\ State-of-Health of Li-Ion Battery Estimation Based on the Efficiency of the Charge Transfer Extracted from Impedance Spectra
}

\author{
Ahmed Yahia Kallel (D), Viktor Petrychenko and Olfa Kanoun *(D)
}

check for updates

Citation: Kallel, A.Y.; Petrychenko, V.; Kanoun, O. State-of-Health of Li-Ion Battery Estimation Based on the Efficiency of the Charge Transfer Extracted from Impedance Spectra. Appl. Sci. 2022, 12, 885. https:// doi.org/10.3390/app12020885

Academic Editor: Yong Nam Jo

Received: 13 December 2021

Accepted: 11 January 2022

Published: 16 January 2022

Publisher's Note: MDPI stays neutral with regard to jurisdictional claims in published maps and institutional affiliations.

Copyright: (c) 2022 by the authors. Licensee MDPI, Basel, Switzerland. This article is an open access article distributed under the terms and conditions of the Creative Commons Attribution (CC BY) license (https:// creativecommons.org/licenses/by/ $4.0 /)$.

\begin{abstract}
Measurement and Sensor Technology, Chemnitz University of Technology, 09111 Chemnitz, Germany; ahmed-yahia.kallel@etit.tu-chemnitz.de (A.Y.K.); viktor.petrychenko@s2014.tu-chemnitz.de (V.P.)

* Correspondence: olfa.kanoun@etit.tu-chemnitz.de
\end{abstract}

\begin{abstract}
Several studies show that impedance spectroscopy is a suitable method for online battery diagnosis and State-of-Health ( $\mathrm{SoH})$ estimation. However, the most common method is to model the acquired impedance spectrum with equivalent circuits and focus on the most sensitive parameters, namely the charge-transfer resistance. This paper introduces first a detailed model of a battery cell, which is then simplified and adapted to the observable spectrum behavior. Based on the physical meaning of the model parameters, we propose a novel approach for $\mathrm{SoH}$ assessment combining parameters of the impedance spectrum by building the ratio of the solid electrolyte interphase (SEI) resistance to the total resistance of SEI and the charge transfer. This ratio characterizes the chargetransfer efficiency at the electrodes' surfaces and should decrease systematically with SoH. Four different cells of the same type were cycled 400 times for the method validation, and impedance spectroscopy was performed at every 50th cycle. The results show a systematic correlation between the proposed ratio and the number of cycles on individual cell parameters, which build the basis of a novel online method of $\mathrm{SoH}$ assessment.
\end{abstract}

Keywords: electrochemical impedance spectroscopy; lithium batteries; State-of-Health; charge transfer; solid electrolyte layer

\section{Introduction}

State-of-Health (SoH) defines the usability of the battery for an application and plays a crucial role in the accurate estimation of the remaining state-of-charge (SoC) of a battery. An erroneous SoH estimation results in overestimation of the better capability, as well as a false state-of-charge indication, eventually leading to premature system shutdowns. Furthermore, an unhealthy or aged battery has a lower charge/discharge efficiency and increased heat dissipation, which can cause thermal runaways and explosions if not treated seriously.

The term "State-of-Health" is subjective, as it describes the battery's condition in general. Most studies in the literature associate the SoH of the battery with either a capacity fade, inferring mainly a decrease in the charge storage ability, or a power fade, inferring primarily the increase of impedance limiting the extractable power, or associated with both [1-4]. An insight into already existing methods for evaluating the SoH using either power or capacity fade is provided in Section 2. These methods are grouped into experimental and adaptative methods. Experimental methods are very accurate [4,5]. However, most of the methods discussed in the literature (see Section 2) address individual cell parameters and must therefore be tuned or reconfigured to fit to other cells, even if they have the same type. Other proposed methods in the literature require end-of-life parameters to calculate the current aging state.

In this paper, we propose a SoH assessment method based on impedance spectroscopy for the online diagnosis of battery cells, which could be applied without needing precycling information or being tuned by considering individual cell parameters. The aim is 
to realize a robust and practicable method with low complexity for SoH assessment based on measurements of the impedance spectrum.

Typically, experimental methods for SoH assessment based on impedance spectroscopy fit equivalent circuits to the impedance spectra and correlate the most SoH sensitive equivalent circuit parameters, mainly the charge transfer's equivalent resistance, doublelayer capacitance, or SEI layer, or all of them to the number of charging cycles.

In this paper, we follow a different approach based on a detailed model of the spectrum of a battery cell describing the positive and negative electrodes together with the SEI layer. After model simplification and adaptation to the observable spectrum behavior, we look for a metric combining model parameters in a deterministic manner, which provides information about $\mathrm{SoH}$.

The paper is structured in six sections. In Section 2, an overview of battery structure, the effect of aging, and the main reasons for power fade and capacity fade. Section 3 contains the experimental details used to conduct the cycling and the impedance spectroscopy. Section 4 includes the proposed solution to estimate the SoH. Finally, Section 5 contains the IS data results, modeling, and analysis, in addition to applying the proposed method to extract the $\mathrm{SoH}$.

\section{State of the Art}

Several methods have been reported to observe, monitor, or estimate the $\mathrm{SoH}$. These methods can be classified into two main groups: The experimental methods, which are based on direct measurements or models based on actual measurements, and the adaptive methods, which try to simulate or estimate the health of the battery provided the behavior of the battery and minimal measurement data [5]. These methods are shown in Figure 1.

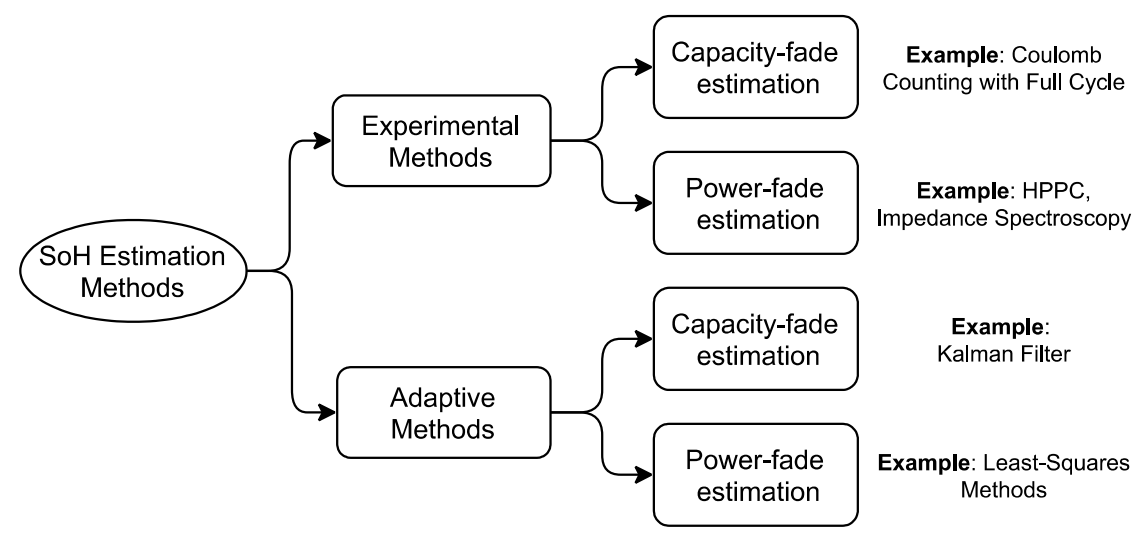

Figure 1. State-of-the-art methods for State-of-Health (SoH) estimation.

Experimental methods are based on results of experimental investigations carried out before the real life of the battery cell, which serve as a basis for estimation of SoH. They can be classified into capacity-fade estimation methods and power-fade methods. Experimentalbased capacity-fade assessment methods, such as Coulomb-counting, measure the battery's capacity. With the load disconnected and the battery integrally cycled, the total charge, inferred from the integration of the current over time, gives the battery's actual capacity. The main inconvenience with this method is that the measurement over a complete cycle, including a full charge and discharge process, is time-consuming and involves high energy consumption. Nonetheless, it is the standard for determining the battery capacity.

Experimental power-fade estimation methods are mainly based on impedance-based information. Among these techniques is the Hybrid Pulse Power Characterization (HPPC), which uses a current pulse, which causes a sudden voltage to drop or rise depending on the sense of the current. The current and voltage information is used to calculate the charge or discharge power [6]. A significant drawback of this method is its heavy reliance on the series resistance, which is heavily influenced by contact resistance, which is often sensitive 
to electronic noise and environmental influences, including temperature and electrical noise. Using a multifrequency current excitation signal, and with the help of the response voltage, an impedance spectrum (IS) can be extracted. This gives more information about the battery's performance in different frequencies and is not limited to the DC component, such as in the HPPC method. Furthermore, the obtained spectrum is less sensitive to noise and environmental factors. This is due to the quality of the signal processing required for such a task and the consistency of the measurement points owing to the Kramers-Kronig relation. However, a major drawback is the necessity of an equivalent circuit model (ECM) to deconvolute the impedance spectrum and extract one or more sensitive parameters to SoH. The choice of the model, as well as the parameters, affects the eventual results. After fitting the measurement to an equivalent circuit model, the obtained circuit parameters (e.g., resistance), or their properties (e.g., constant-phase angle), are correlated with the $\mathrm{SoH}[4,5]$. In [7], a resistance in series with an inductance and $2 \mathrm{RQ}$ elements is used to model the behavior of the electrodes and electrolyte. The series resistance and one electrode's resistance are correlated with the number of cycles. In [8], an Rs-L-Randles circuit is used to model the battery. A formula estimating the battery capacity from the series resistance is used.

Similarly, in [9], the same model, including a Warburg element, is used. The growth rate of the charge transfer of one of the electrodes shows a high correlation with the number of cycles. The paper features a ratio of SoH that requires the current and the end-of-life charge-transfer resistance to be calculated. In [4], the battery is modeled with the help of a resistance in series with an inductance and RC element, plus a transmission line model (TLM). Here, TLM depicts the transmission line model to describe the electrode. Despite the complexity of the model, the paper shows a correlation between several components, including charge-transfer resistance, electrode capacitances, to the number of cycles. In [10], a simplified RC element is used for the fitting. The model uses a linear combination of the sum of the resistance of electrodes and charge transfer and the double-layer capacitance to predict the end-of-life values. In [11], a model consisting of a resistance in series an inductance, a Warburg element, R/ / L, and 3RCs is used. The series resistance is found to be the most correlated with the SoH. In [12], an exponential regression model based on the SEI layer resistance from an ECM fitting with 3RQ in series with Warburg element and resistance is depicted. Ref. [13] contains a detailed review of SoH prediction methods based on impedance and impedance spectroscopy. This review shows that most literature directly uses the impedance spectrum, or a direct value of the ECM, which is then correlated with the capacity or number of cycles. Most of the state-of-the-art methods based on impedance spectroscopy have demonstrated a good correlation with the $\mathrm{SoH}$, which gives high accuracy greater or equal to 95\% [5]. However, they use cell-dependent values for the correlation or linearized with future values, making generalizing the method even for the same battery types unfeasible.

Adaptive methods use mathematical models based on current and voltage measurements during operation to estimate the capacity or the impedance. They can be classified into capacity-fade estimation methods and power-fade methods. Adaptive capacity-fade estimation methods are inferred by an online current and voltage measurement and mainly involve estimation theory (e.g., filters-based approaches). For example, the Kalman filter and its derivatives are used to either estimate the total capacity in steady white noise filtering configuration $[6,14,15]$ or to estimate the power decay by estimating the internal resistance from running signals [16]. Least-square methods, such as Ordinary Least Squares, Weighted Ordinary Least Squares, and Total Least Squares, have also been used for capacityfade detection. One possible implementation is estimating a Coulombic efficiency-like quantity over time and aging, which is compensated inside the total capacity [6].

Adaptive methods are argued to have outstanding accuracy $[5,6]$, but they require a long calibration/estimation time for reaching high accuracy. They also have considerable risks of deviation after a long-running time as their principle is mainly incremental, and deviations are therefore accumulated. 
Experimental methods are often thought to be limited due to measurement deviations or difficulties of interpretation and end-of-life estimation. For example, HPPC does not provide accurate results if the batteries were not at rest before measurement or when the cells are affected by environmental factors, e.g., temperature and noise. Impedancespectroscopy-based methods are more reliable, as they rely on several measurement points. Although they are not prone to noise, it is possible to use curve fitting to filter them out. Moreover, the impedance spectrum is very sensitive to SoH. However, a significant problem is a dependency on the cell's individual properties. The measurements tend to be very individual and may even fail to provide a generalizable solution to be applied to cells from the same type or same manufacturer. Although other solutions have investigated the linearization of the fitted circuit elements to future end-of-life values, such as in [4], these solutions may not be realizable in reality.

In this paper, we investigate the possibility of providing a linearization function to calculate the SoH that is less affected by the cell's individual properties. The proposed solution aimed to work individually but can also be incorporated as a basis for adaptative solutions $[1,5,6]$.

\section{Theoretical Background}

A lithium-ion battery cell has a negative electrode, commonly made of graphite, and a positive electrode based on $\mathrm{Li}^{+}$. Both are separated by an electrolyte and a separator (see Figure 2). Electrons are transported from one electrode to the other during charge or discharge processes through the external circuit. Inside the battery cell, this current is compensated by ions movement in two steps: Dissolution of $\mathrm{Li}^{+}$in the electrolyte from the emitting electrode and rejoining a $\mathrm{Li}^{+}$from the electrolyte with the electron coming from the external circuit to form $\mathrm{Li}$ in the receiving electrode [1].

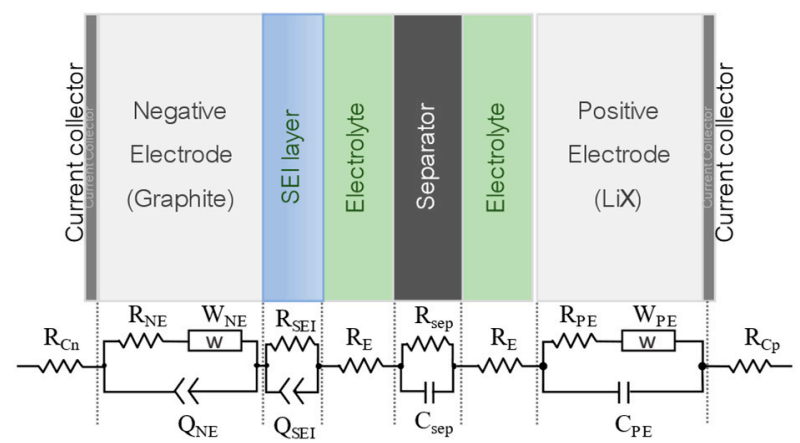

Figure 2. Decomposition of a battery impedance spectrum.

During the battery's first cycles, a solid electrolyte interphase (SEI) layer is formed by inactive lithium due to electrolyte decomposition over the negative electrode. The SEI continues to grow, affecting both capacity and power to fade. Moreover, several pores will be filled by covering the negative electrodes, causing more power to fade [6,17]. Among other reasons for negative electrode aging are metallic lithium plating, decomposition of the binder, the loss of contact of active material particles due to a volume change, which forms capacity fade. Similar aging is perceivable in the positive electrode [17]. In summary, most of the aging is caused by lithium ions stalling.

The mechanism within a battery cell and the construction of the battery itself could be described with the help of a lumped equivalent circuit model (ECM), as shown in Figure 2. A detailed ECM impedance model is elaborated in this figure based on $[1,18]$. The positive electrode can be modeled with a Randles circuit $\left(R_{P E}, C_{P E}\right.$, and $\left.W_{P E}\right)$ [18]. $R_{P E}$ is the charge-transfer resistance within the positive electrode and can be described using a Nernst equation as the electrons (de)intercalating the positive electrode to/from the electrolyte. It is inversely proportional to the number of electrons $\mathrm{n}_{\mathrm{ct}, \mathrm{PE}}$ involved in the charge transfer in 
the positive electrode, and the exchange-current density $i_{0, c t}$ as depicted in [19] and shown in Equation (1):

$$
\mathrm{R}_{\mathrm{PE}}=\frac{\alpha_{\mathrm{ct}}}{\mathrm{n}_{\mathrm{ct}, \mathrm{PE}}}
$$

where $\alpha_{\mathrm{ct}}=\frac{\mathrm{RT}}{\mathrm{Fi}_{0, \mathrm{ct}}}$ is a constant [19] which contains the universal gas constant (R), the temperature in Kelvin (T), and the Faraday constant $(\mathrm{F}) . \mathrm{C}_{\mathrm{PE}}$ is the double-layer capacitance to model the charge difference between the electrolyte and the positive electrode. $W_{\mathrm{PE}}$ is the diffusion process to the electrode. Similarly, the negative electrodes share the same Randlesalike structure $\left(\mathrm{R}_{\mathrm{NE}}, \mathrm{Q}_{\mathrm{NE}}\right.$, and $\left.\mathrm{W}_{\mathrm{NE}}\right)$. Due to the pores inside the negative electrode during the constructions, the capacitor is "imperfect" and is often substituted with a constantphase element named $\mathrm{Q}_{\mathrm{NE}}$. In most lithium-ion cells, separating the charge transfer within positive and negative electrodes using impedance spectroscopy is not feasible without accessing internal battery cell components, and a composite electrode model can be instead considered [4]. In this case, the lumped ECM of the charge transfer is as described in Equation (2):

$$
\mathrm{R}_{\mathrm{ct}}=\frac{\alpha_{\mathrm{ct}}}{\mathrm{n}_{\mathrm{ct}}}
$$

here $\mathrm{n}_{\mathrm{ct}}$ is the charge-transfer electrons involved in the charge-transfer phenomena and $\mathrm{i}_{0}$ is the exchange current.

Next is the separator, which is often viewed as a non-conductive $R C$ circuit $\left(R_{\text {sep }}, C_{\text {sep }}\right)$. The electrolyte is modeled as a resistance $\left(R_{E}\right)$. Finally, the side reaction, which consists of lithium plating and SEI layer, and mainly represented as a thin film on top of the positive and negative electrodes, which are more impacted in the "SEI layer", can be described by an RQ circuit $R_{S E I}$ and $Q_{S E I}$ considering both of them are a reduction reaction. Here $\mathrm{R}_{\mathrm{SEI}}$ could be expressed in terms of the constant divided by the electron concentration $\mathrm{n}_{\mathrm{SEI}}$ within the SEI layer, as shown in Equation (3):

$$
\mathrm{R}_{\mathrm{SEI}}=\frac{\alpha_{\mathrm{SEI}}}{\mathrm{n}_{\mathrm{SEI}}}
$$

where $\alpha_{\mathrm{SEI}}=\frac{\mathrm{RT}}{\mathrm{Fi}_{0}, \mathrm{sei}}$. Similar, $\mathrm{i}_{0, \mathrm{SEI}}$ is the exchange-current density in the side reactions.

\section{Proposed Method}

Conventionally, in impedance spectroscopy, the values of the parameter of an equivalent circuit after parameter extraction are represented versus the SoH. The ECM parameter showing the highest dependence on $\mathrm{SoH}$ is chosen for its assessment. Due to the random development of the side reactions in the cells related to the operation parameters, the evolution of the different elements of the cell gives each cell a unique development. As discussed in the previous section, the mechanisms inside a cell are still the same, but they influence the electrical properties of the cells differently due to the unique SEI layer characteristics gained during the first cycles.

The same behavior can be observed for the charge transfer, as fewer electrons would still be active in the process [17]. In this case, the resistance of charge transfer, or the resistance inferred by trapped electrons in SEI, is one of the most prominent for $\mathrm{SoH}$ estimation and is mainly used in the literature. However, it is not a generalized solution, which could be applied to all the cells, even from the same manufacturer.

Using a ratio of the electrons involved in the charge transfer and comparing them to the electrons stalled in the SEI layer, it is possible to infer the SoH pragmatically. Unfortunately, accessing these parameters is not directly feasible. However, an image of the electrons can be provided from the charge transfer and SEI resistances following an appropriate ECM. Therefore, following a data fitting, the resistances can infer the electrons' images, as shown in Equation (4). Using the ECM provided in the next section and enforcing a current, 
and assuming that the current at low frequencies passes through the resistances only, the approximation Equation (4) becomes valid:

$$
\eta_{\mathrm{ct} / \mathrm{SEI}}=\frac{\mathrm{R}_{\mathrm{ct}}}{\mathrm{R}_{\mathrm{ct}}+\mathrm{R}_{\mathrm{SEI}}}=\frac{\mathrm{n}_{\mathrm{ct}} \alpha_{\mathrm{SEI}}}{\mathrm{n}_{\mathrm{SEI}} \alpha_{\mathrm{ct}}+\mathrm{n}_{\mathrm{ct}} \alpha_{\mathrm{SEI}}} \propto \frac{\mathrm{n}_{\mathrm{ct}}}{\mathrm{n}_{\mathrm{SEI}}+\mathrm{n}_{\mathrm{ct}}}
$$

Experimental investigations in the following section have the aim to validate this theoretical and chemical-physical-based hypothesis. Therefore, it is worth investigating if this method depends on individual cell parameters, which would require a priori experimental measurements and calibrations.

\section{Hardware Setup}

This section focuses on collecting measurement data based on the cycling of battery cells and measuring impedance spectra over many cycles.

First, four commercial Li-ion cells of type Sony US18650VT3 have been cycled 400 times. Each cell is charged and discharged using Constant-Current Constant-Voltage (CCCV) with a rate of $1 C(1.5 \mathrm{~A})$ using an embedded device ICharger106+. Between each cycle, 15 min of relaxation time were assured. To ensure a charge balance between the cells, passive balancing is fulfilled every 20 cycles. An example of the voltage and current profile during the CCCV charging procedure is shown in Figure 3.

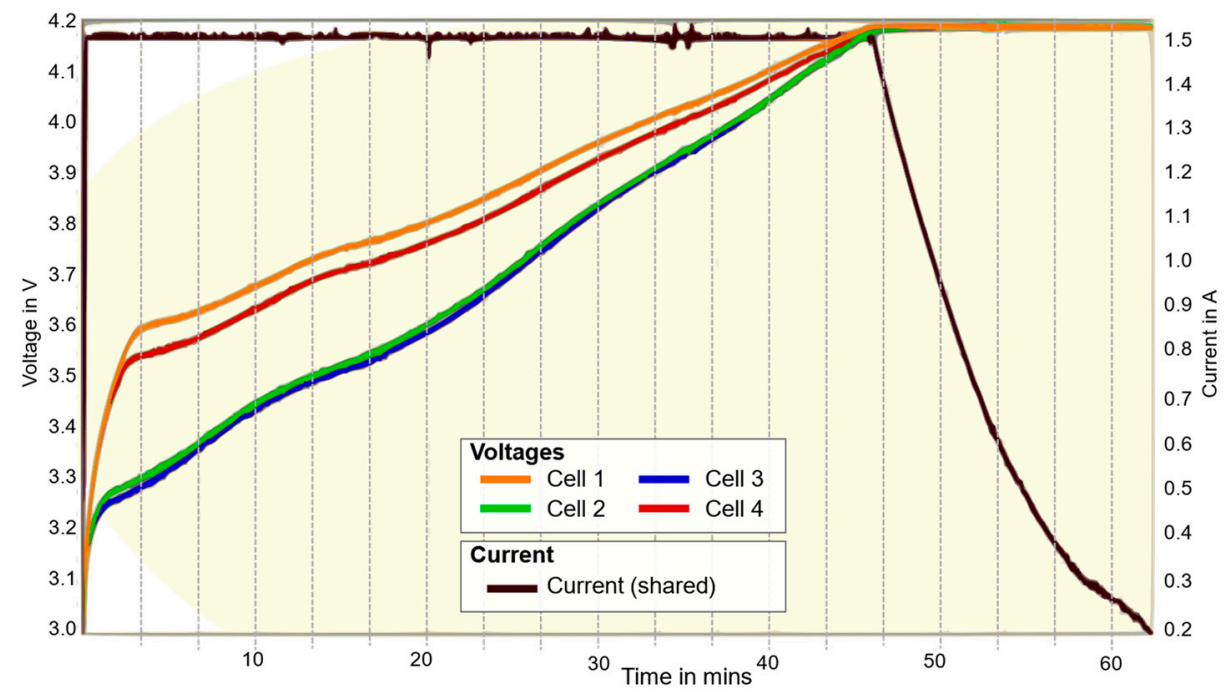

Figure 3. Voltage and current profile of the 4 cells, during CCCV charge.

Afterward, and at every 50 cycles, impedance spectroscopy is carried out for each cell. The impedance spectroscopy is done using a compact Data Acquisition system (NI cDAQ 9881), a custom-made control circuit, a voltage-controlled current source (VCCS) based on Servowatt DCP 130, and a computer running a custom LabVIEW script. A simplified measurement block is shown in Figure 4. Here, a sinewave-based current excitation signal is injected into the battery, which is afterward, together with the response voltage signal from the battery, sampled using an ADC module in the NI-cDAQ9881. The system can measure the impedance spectrum of 4 cells in series, with 81 logarithmically distributed frequencies within the frequency range of $10 \mathrm{mHz}$ to $1 \mathrm{kHz}$. To filter the noise and enhance the signal, the final impedance spectrum measurements are processed using MATLAB. 


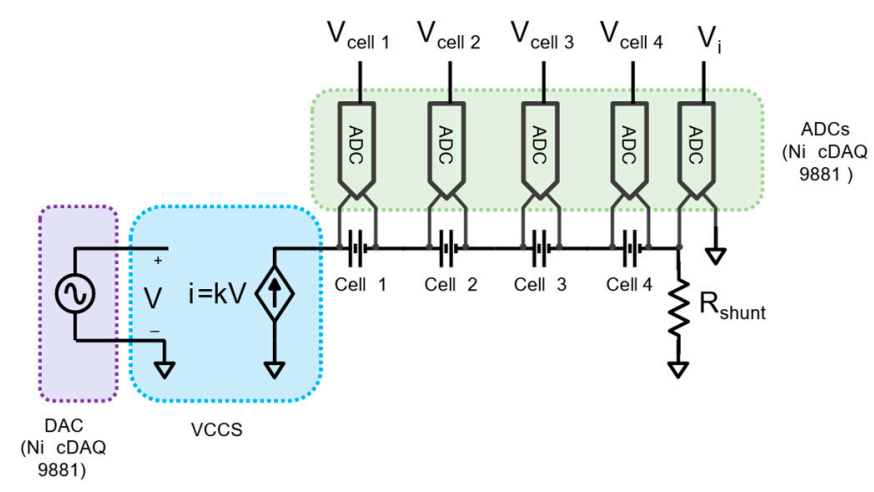

Figure 4. Flowchart of the cycling and measurement procedure.

The cycling and impedance spectroscopy are repeated until the 400th cycle is reached. A complete overview of the workflow can be found in Figure 5.

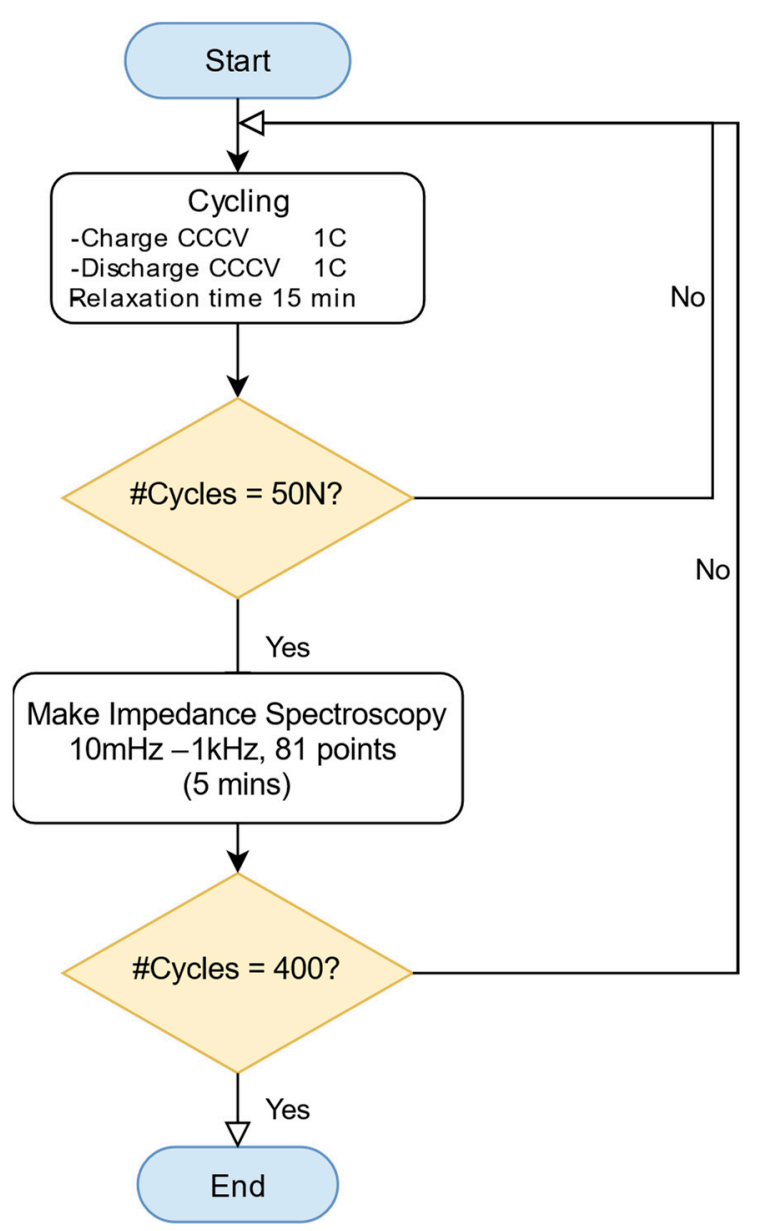

Figure 5. Flowchart of the cycling and measurement procedure.

\section{Results and Analysis}

After 400 cycles, with a step of 50, eight impedance spectra are obtained per cell. The obtained measurement spectra of the battery (example of the first) cell show a global increase over the cycles (see Figure 6). Furthermore, it is shown in Figure 7 that cell one and cell four, respectively cell two and cell three, have a similar cell voltage profile after 400 cycles. 


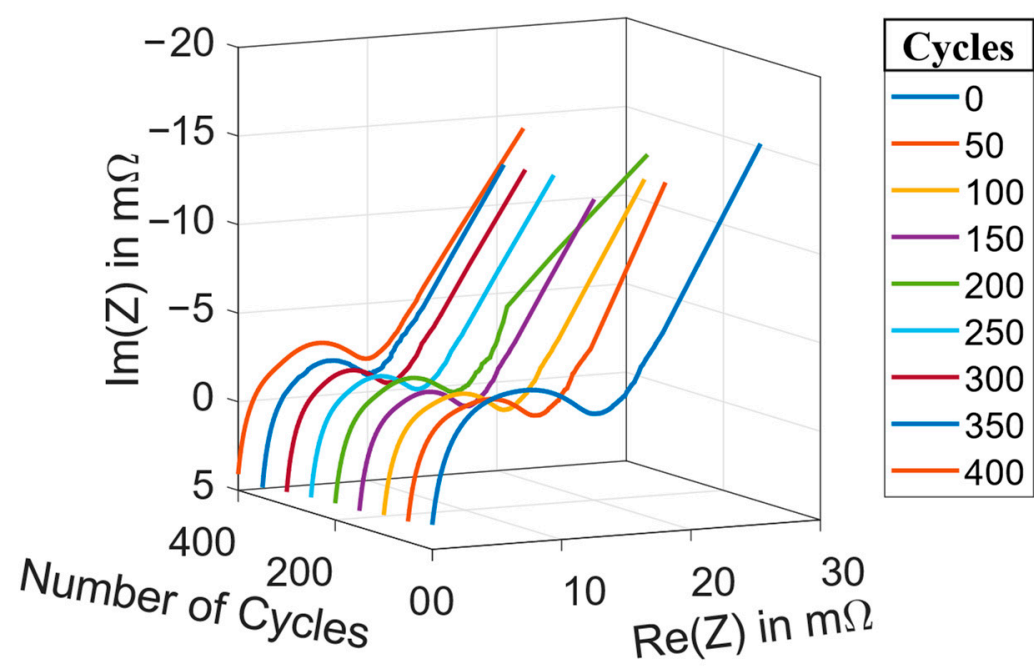

Figure 6. Impedance spectrum measurements for cell one as a function of the number of cycles.

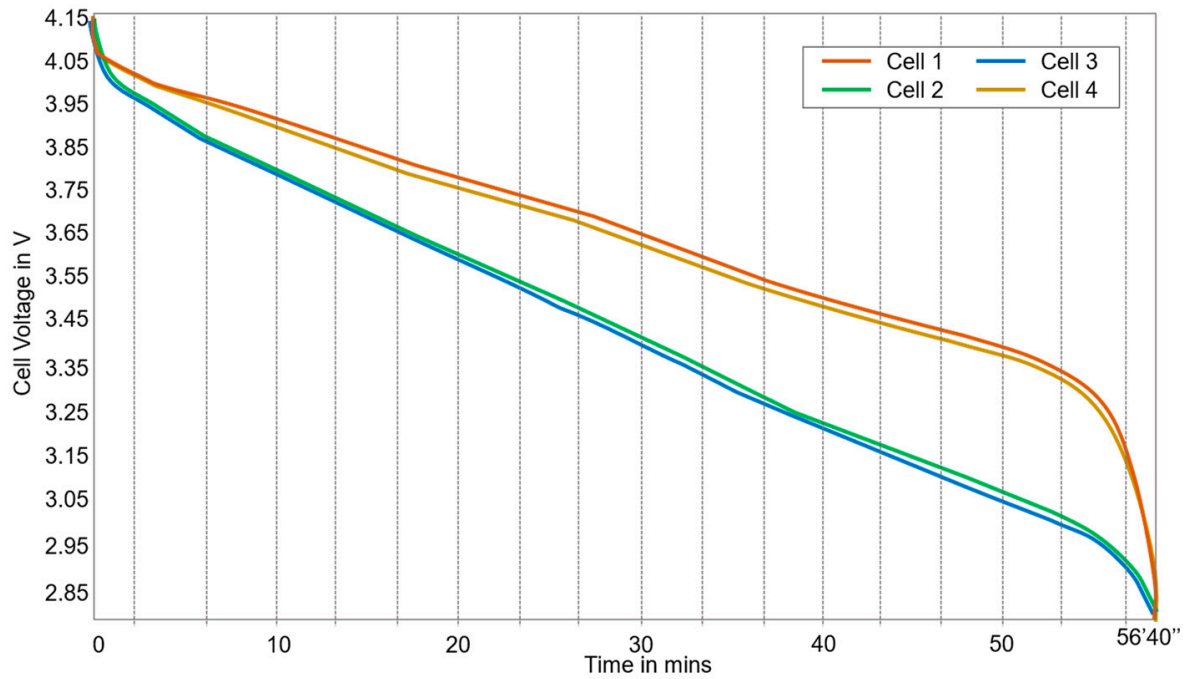

Figure 7. Voltage discharging profile at the 400th cycle.

\subsection{Qualitative Impedance Spectrum Analysis}

In this subsection, we focus mainly on the qualitative analysis of the impedance spectrum of a single cell at a single cycle. Figure 8a shows an example impedance spectrum obtained for cell 1 at cycle 150. The general form and behavior stay the same for each cell at different cycles.

The impedance spectrum in Figure 8a shows that not all the expected mechanisms in the battery could be measured. However, a clear pattern can be discerned depending on the region: The low frequencies (right side of the figure) show a diffusion process combined with a charge transfer pattern corresponding to one or both electrodes. Middle frequencies (middle of the figure) show a semi-circle on the Nyquist curve, corresponding to either SEI or separator. Finally, the high frequencies (left side of the figure) show a resistive-inductive part, influenced by current conduction due to cylindrical cell geometry. 


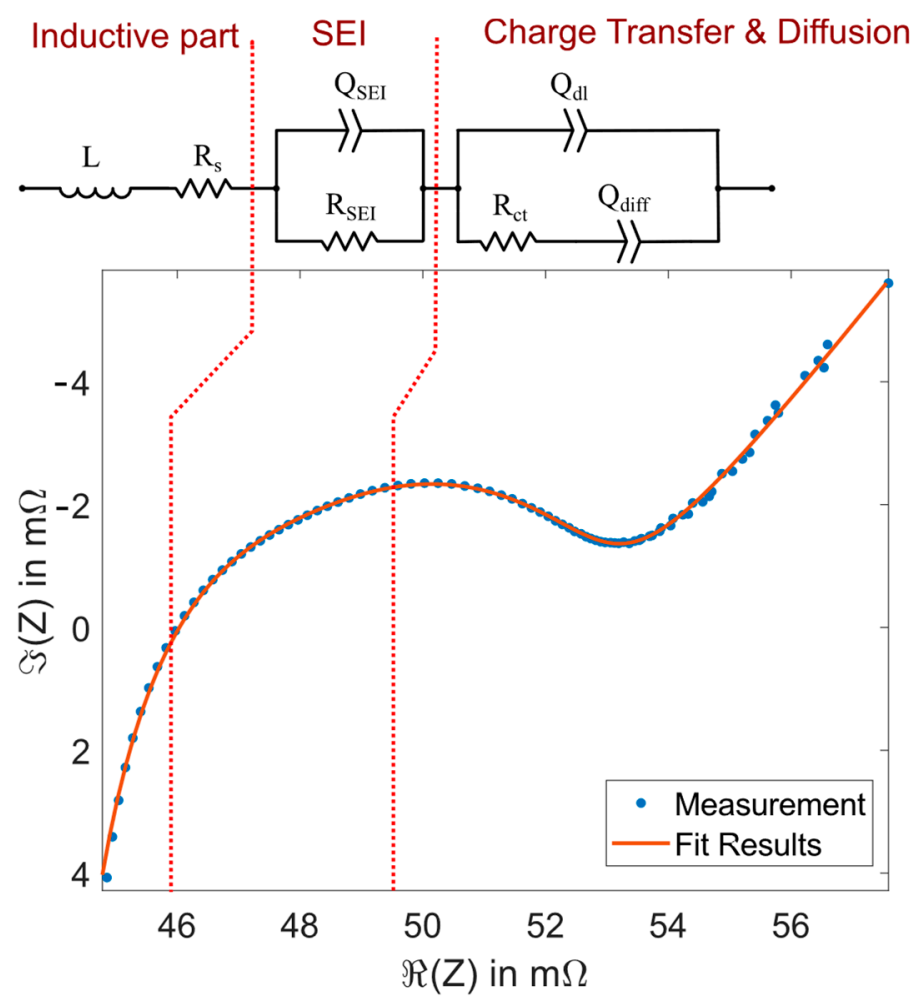

(a)

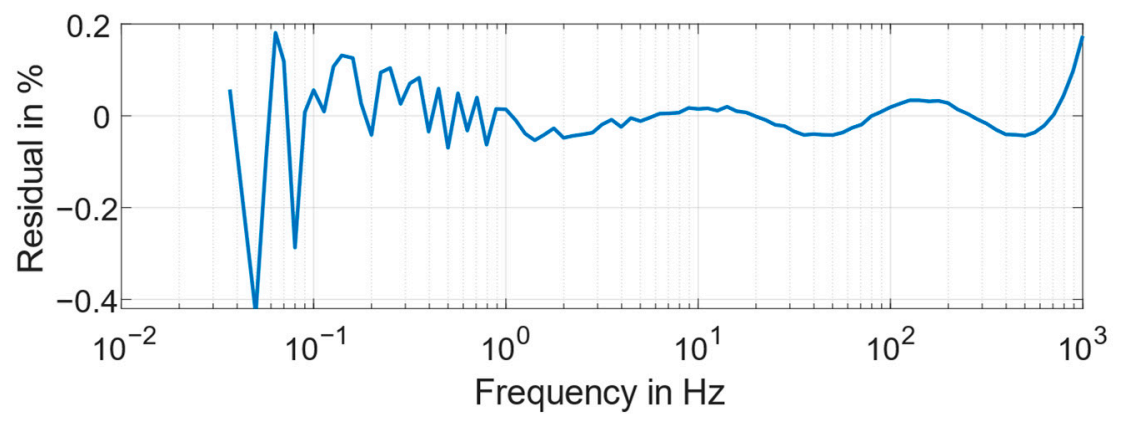

(b)

Figure 8. (a): Fitting result of the ECM model to the impedance spectrum of cell one, cycle 150 and (b): The residual of the fitted ECM model to impedance spectrum of cell one, cycle 150.

The ECM proposed in Figure 8a is used to model the behavior of the impedance spectrum: A combined electrodes' behavior described by a modified Randles Model is used to model both electrodes. In this case, the double-layer capacitor is changed to CPE element $\mathrm{Q}_{\mathrm{dl}}$, and the Warburg element is replaced with a CPE $\mathrm{Q}_{\mathrm{diff}}$, to account for diffusions from both electrodes. The separator is combined with the SEI, and Here the SEI is modeled as an $R Q$ element. Finally, a series resistance $\left(R_{S}\right)$ and an inductance $(L)$ are used to model the conductive part inside the battery and the electrolyte resistance.

The fitting results show high similarity between the measured spectrum and the fitted spectrum, as shown by the example fitting in Figure 8a. Furthermore, a qualitative residual analysis based on the Equation (5) shows that the fitting had less than $0.4 \%$ relative deviation.

$$
\widetilde{Z}_{\text {rel }}=\frac{\left|Z_{\text {meas }}\right|-\left|Z_{\text {fit }}\right|}{\left|Z_{\text {meas }}\right|}
$$

By closely examining the residual curve (see Figure $8 b$ ), the fitting seems to be subject to systematic deviations. The quantization error affects the low frequencies due to the limited capability of the measurement device [20]. The middle frequencies and high 
frequencies show a systematic behavior in the residuals with an oscillating pattern. The oscillation corresponds to model deviations, which principally show the need for model extensions. This effect can be neglected for the investigation, as the residual amplitude is sufficient and less than $0.4 \%$.

\subsection{Post-Fitting Parameter Analysis}

The results of the post-fitting of the proposed equivalent circuit are shown in Figure 9. The double-layer capacitance and charge-transfer resistance, as well as SEI, are most influenced by aging. Here $R_{\mathrm{ct}}$ increases, while $\mathrm{R}_{\mathrm{SEI}}$ slightly decreases as a function of the number of cycles for all the battery cells. The parameters of the IS show a systematic behavior but are pretty different from one cell to another, even if the cells are of the same type.
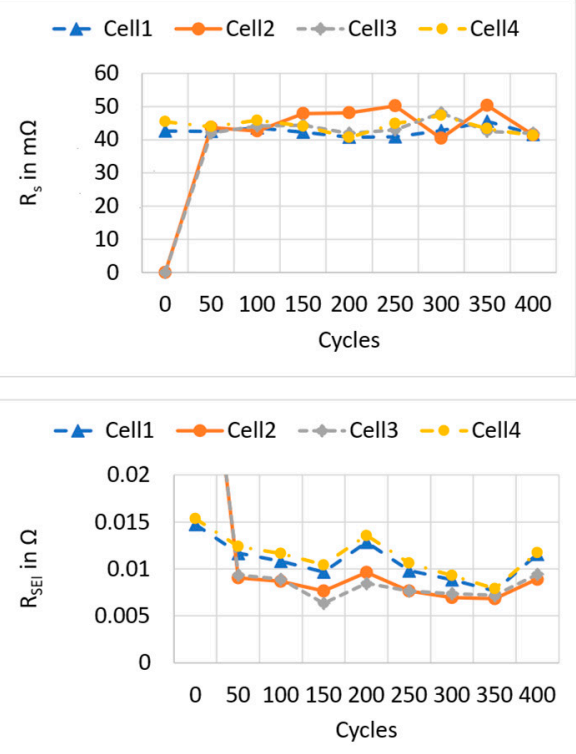

$-\leftarrow$ Cell1 $\rightarrow$ Cell2 $-\nleftarrow-$ Cell3 $-\bullet-$ Cell4
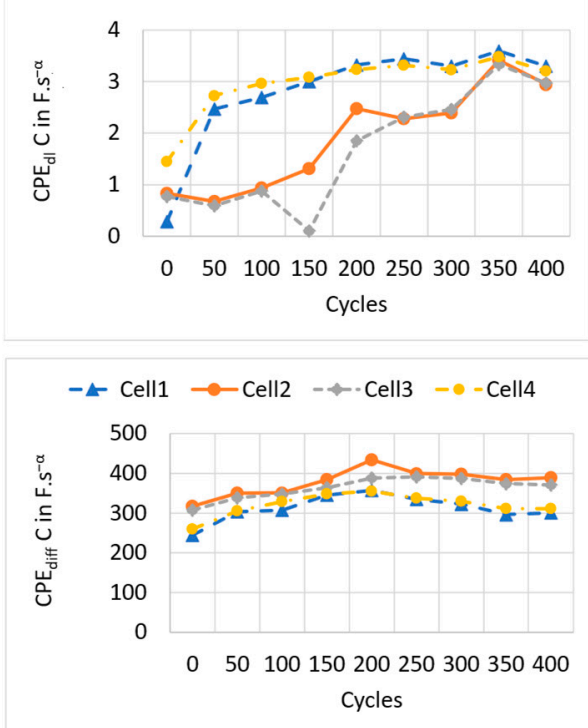

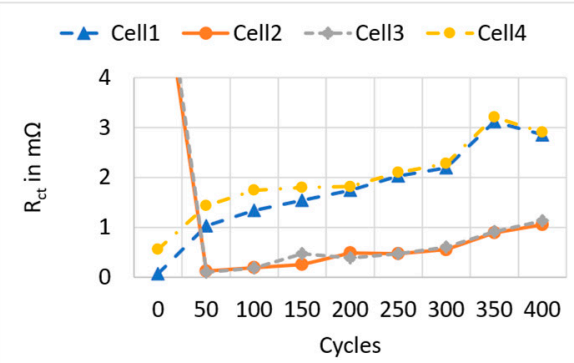

- Cell1 $\rightarrow$ Cell2 $-\diamond$ Cell3 $-\bullet-$ Cell4

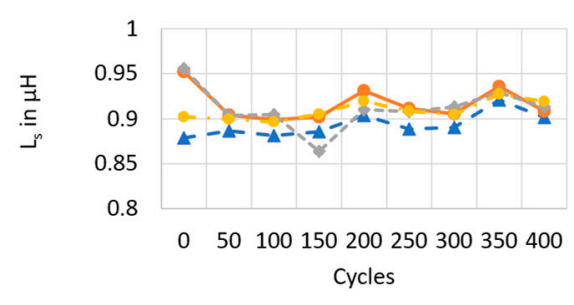

$-\leftarrow$ Cell1 $\rightarrow$ Cell2 $-\leftarrow$ Cell3 $-\bullet-$ Cell4

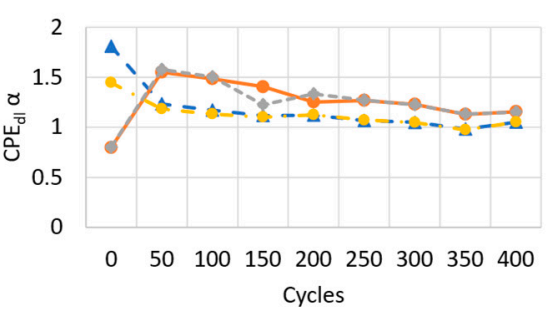

$-\leftarrow$ Cell1 $\rightarrow$ Cell2 $-\leftarrow$-Cell3 $-\bullet-$ Cell4

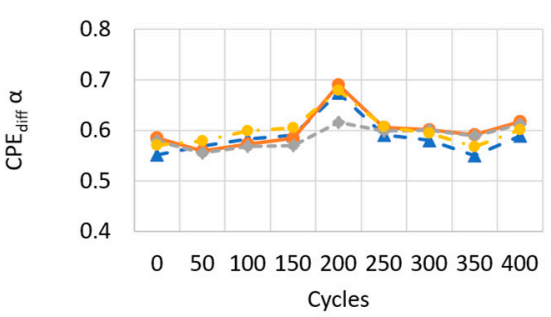

Figure 9. Cont. 

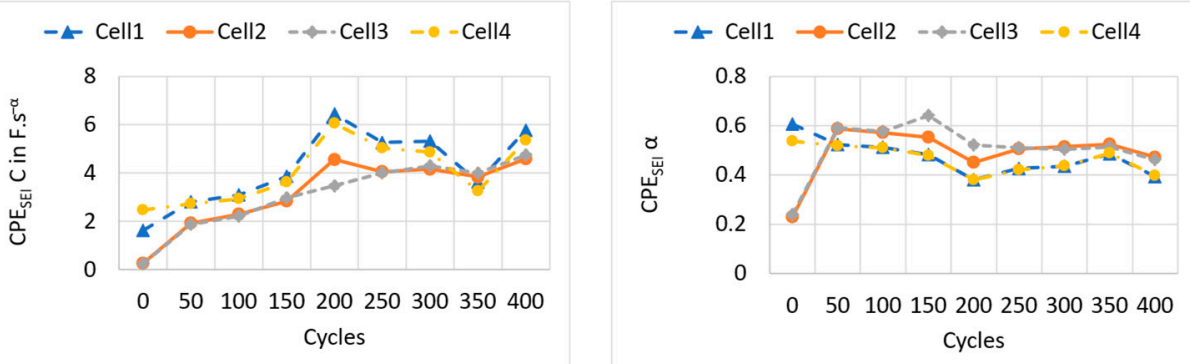

Figure 9. Data fitting results of all the ECM components.

By inspecting cell 1 in detail: The series contact resistance $R_{S}$ shows a slight increase with a coefficient of $35 \mu \Omega$ per cycle, as shown in Figure 10a. The charge-transfer resistance $R_{c t}$ increases as fewer electrons are involved in this mechanism, and more electrons are trapped in the SEI layer. A linear fit shows a slight rise in $R_{\mathrm{ct}}$ with a rate of $0.32 \mathrm{~m} \Omega$ per cycle. This increase is accompanied by a decrease in $R_{S E I}$ with a rate of $0.47 \mathrm{~m} \Omega$ per cycle, as shown in the linear fit of $\mathrm{R}_{\mathrm{SEI}}$ in the same figure.

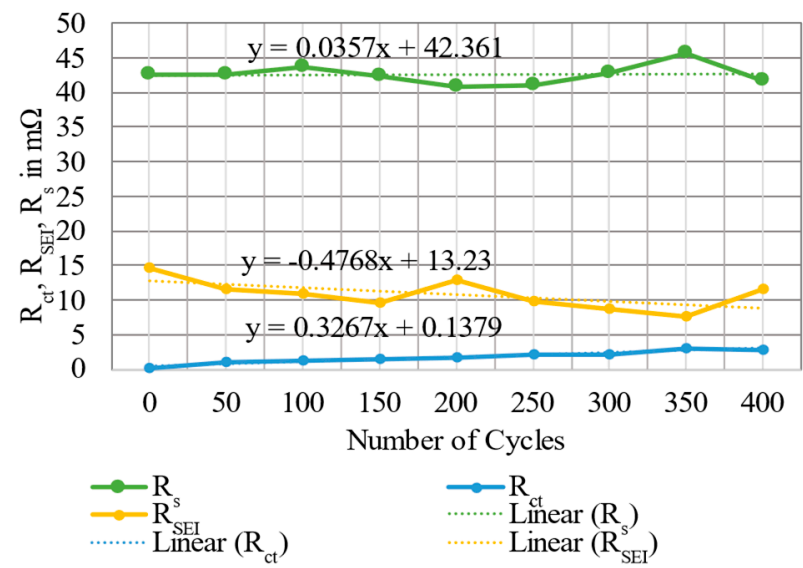

(a)

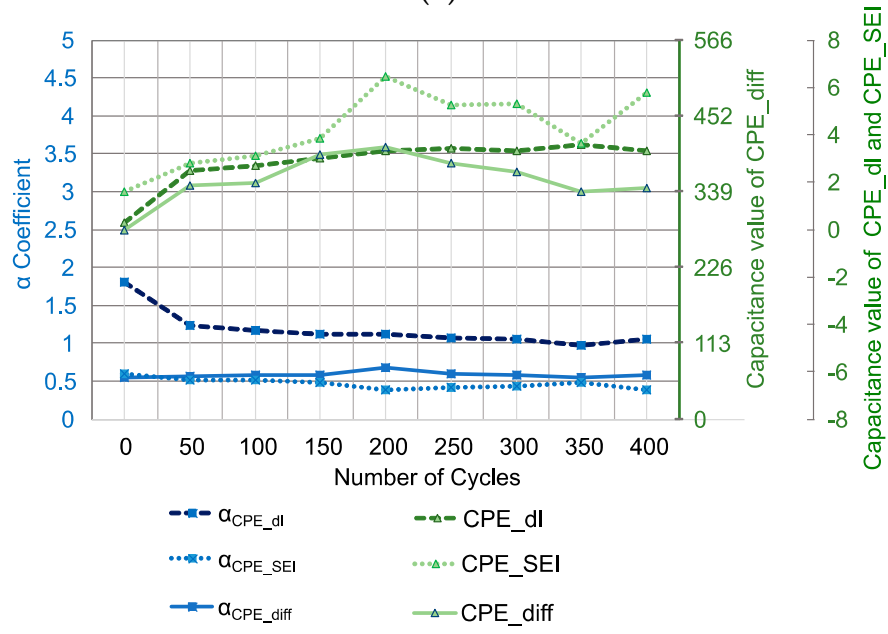

(b)

Figure 10. (a) Analysis results of $R_{s}, R_{c t}$, and $R_{S E I}$ for cell one as a function of the number of cycles, (b) Analysis results of parameters of $Q_{\mathrm{dl}} \mathrm{Q}_{\mathrm{SEI}}$ and $\mathrm{Q}_{\mathrm{diff}}$ for cell one as a function of the number of cycles.

The fitting results in Figure 10b show the correlation of the parameters to the $\mathrm{SoH}$. Therefore, the double-layer CPE (CPE_dl) shows the highest dependence on the $\mathrm{SoH}$, but 
this dependence is non-linear. Furthermore, its influence dominates at low frequencies, which are affected by systematical deviations. This deviation heavily influences other parameters, namely the alpha-coefficient of the double-layer CPE, the capacitance and alpha-coefficient of the diffusion CPE. On the other hand, the SEI capacitance and its corresponding alpha-coefficient show less dependency on $\mathrm{SoH}$, as shown in Figure 10b. In fact, the diffusion capacitance (CPE_diff), which exponentially increases due to the film layer forming, is analogous to the increase of the Warburg element depicted in [4]. As more electrons deposit on the SEI layer, the capacitance of the SEI layer also increases (CPE_SEI). On the other hand, the alpha coefficients of SEI ( $\left.\alpha_{\mathrm{CPE} \_\mathrm{SEI}}\right)$ and of the diffusion $\left(\alpha_{\mathrm{CPE} \_ \text {diff }}\right)$ are quasi-constant around 0.5. Due to the model which encapsulates both electrodes into one modified Randles circuit, the alpha-coefficient of the double layer $\left(\alpha_{\mathrm{CPE} \_\mathrm{dl}}\right)$ is higher than 1. As more electrons are deposited on the pores of the electrodes, the porosity is shrinking during cycling, which explains the decreasing value of the alpha-coefficient.

Therefore, the consideration of individual model parameters is not sufficient. From all parameters, charge transfer and double layer capacitance show the highest sensitivity to aging. As the resistance values mainly influence the impedance spectrum, they are less prone to model mismatch and tuning problems: They tend to have more linear behavior than the CPE element's capacitance and alpha-coefficient. However, the SoH estimation from the resistances alone cannot be generalized: The parameters are mainly dependent on the batteries shaped during the first cycles of the batteries, making different offsets in $R_{c t} v s$. the number of cycles in figure. Furthermore, the aging coefficient seems to be also dependent on the cells, which makes the slope of $R_{\mathrm{ct}} \mathrm{vs}$. the number of cycles also different, as shown in Figure 9.

To quantify the contributions of electrons in SEI and charge transfer, Equation (4) is applied. Figure 11 shows that the ratio of charge-transfer electrons $R_{c t}$ to total chargetransfer electrons and SEI concentration $\left(R_{c t}+R_{S E I}\right)$ increases with the number of cycles. This is due to the growth of the SEI layer over the cycles. Simultaneously, the ratio of RSEI to $\left(R_{c t}+R_{S E I}\right)$ decreases from $99.52 \%$ to $80.17 \%$ by the 400 th cycle, at an average rate of $0.02 \%$ per cycle, for cell 1 .

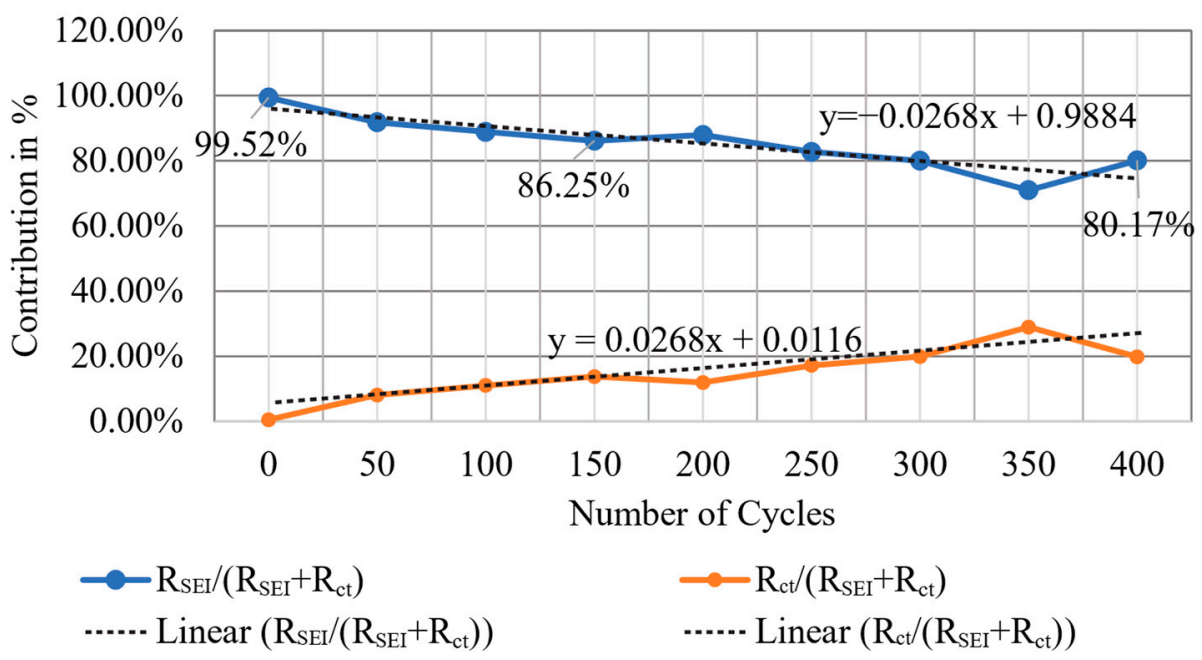

Figure 11. Contribution of RSEI, respectively $R_{c t}$ to $R_{c t}+R_{S E I}$ for cell one as a function of the number of cycles.

By checking the four cells' behavior, the same pattern of the electrons' contribution in charge transfer, corresponding to the contribution of $\mathrm{R}_{\mathrm{SEI}}$ to the total resistances in the charge transfer and SEI layer, can be seen in cells 1 and 4, as shown in Figure 12. Cell 2 and 3 exhibit similar behavior while still decreasing, is still different from cells 1 and 4 . This is due to the fingerprint to each cell, with cells 1 and 4, respectively cell 2 and 3 , sharing a similar cell voltage profile behavior as shown in Figure 6. However, it is also shown that 
this method can only work starting from the 50th cycle, to which the battery's internal chemical architecture is shaped.

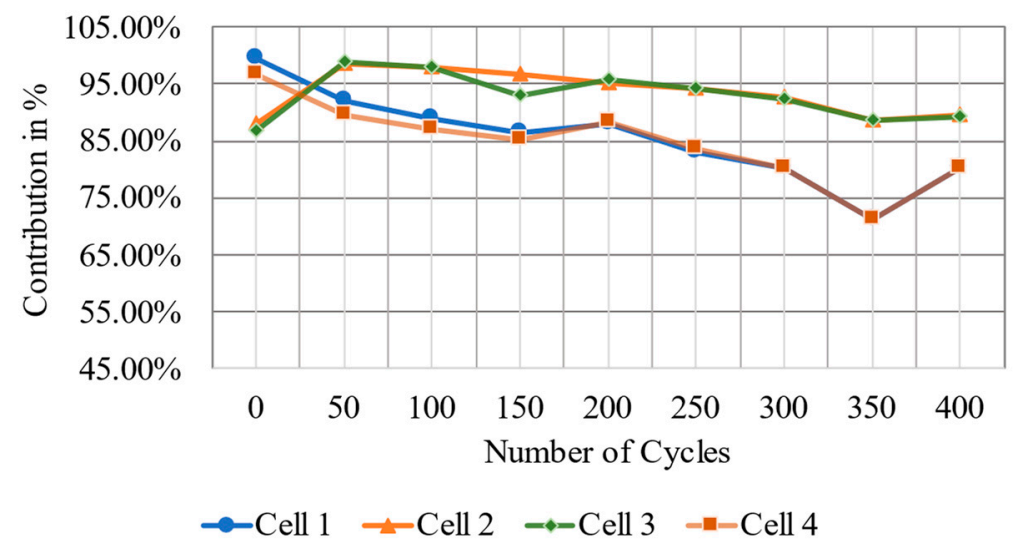

Figure 12. Contribution of $R_{S E I}$ to $R_{c t}+R_{S E I}$ for the four cells as a function of the number of cycles.

Using this method provides the possibility of reliable approximation of SoH without extrapolation to end-of-life, providing an accurate measurement.

\section{Discussion}

The proposed method shows more stability and less sensitivity against noise and quantization errors in the measurement. It also allows for both in-situ and ex-situ measurements, as the contact resistance is estimated beforehand and is not taken into the final SoH estimation calculation. In addition, the proposed method uses a simplified model, which describes the cell's behavior with fewer deviations. Finally, the computational effort required in the curve fitting is less than the TLM-based approach.

In this study, we limited the step of cycles to 50 to emphasize the global behavior of the efficiency of the charge transfer. It is inferred from the fitting in [4] that electrons trapped in SEI could be freed and take part in charge transfer, as the process is random. The effect of this randomness per cycle is the subject of a future study.

\section{Conclusions}

In this paper, we introduced a novel approach combining specific model parameters, based on the ratio of the SEI resistance to the total resistance of SEI and the charge transfer, based on fitting results to a modified Randles circuit, in series with RQ element and a series conductance and resistance for online assessment of $\mathrm{SoH}$. This ratio characterizes the charge-transfer efficiency at the electrodes' surfaces and decreases linearly with SoH at a rate of $0.02 \%$ per cycleover a 400 cycle time course.

Further perspective is to use the efficiency of charge transfer as a feature in artificial intelligence-based algorithms of battery state estimation methods, mainly in BMS control units.

Author Contributions: Conceptualization, O.K.; methodology, A.Y.K.; software, A.Y.K.; validation, V.P. and A.Y.K.; writing-review and editing, A.Y.K. and O.K.; visualization, A.Y.K., V.P.; supervision, O.K. All authors have read and agreed to the published version of the manuscript.

Funding: This research was funded from Bundesministerium für Wirtschaft und Energie, within project "ImpTes-Impedanzbasierter Batterietester zum cloudbasierten Monitoring des Lebenszyklus von Batterien für die Elektromobilität und für Energiespeicher", grant number 03EI3019B.

Institutional Review Board Statement: Not applicable.

Informed Consent Statement: Not applicable.

Data Availability Statement: Not applicable.

Conflicts of Interest: The authors declare no conflict of interest. 


\section{References}

1. Plett, G. Battery Management Systems, Volume I: Battery Modeling; Artech House: Boston, MA, USA, 2015.

2. Wohlfahrt-Mehrens, M.; Vogler, C.; Garche, J. Aging mechanisms of lithium cathode materials. J. Power Sources 2004, 127, 58-64. [CrossRef]

3. Schuster, S.F.; Brand, M.J.; Campestrini, C.; Gleissenberger, M.; Jossen, A. Correlation between capacity and impedance of lithium-ion cells. J. Power Sources 2016, 305, 191-199. [CrossRef]

4. Tröltzsch, U.; Kanoun, O.; Tränkler, H.-R. Characterizing aging effects of lithium ion batteries by impedance spectroscopy. Electrochim. Acta 2006, 51, 1664-1672. [CrossRef]

5. Berecibar, M.; Gandiaga, I.; Villarreal, I.; Omar, N.; van Mierlo, J.; van den Bossche, P. Critical review of state of health estimation methods of Li-ion batteries. Renew. Sustain. Energy Rev. 2016, 56, 572-587. [CrossRef]

6. Plett, G. Battery Management System, Volume II: Equivalent-Circuits Methods; Artech House: Nowood, MA, USA, 2016.

7. Stroe, D.I.; Swierczynski, M.; Stan, A.I.; Knap, V.; Teodorescu, R.; Andreasen, S.J. Iagnosis of lithium-ion batteries state-of-health based on electrochemical impedance spectroscopy technique. In Proceedings of the 2014 IEEE Energy Conversion Congress and Exposition (ECCE), Pittsburgh, PA, USA, 14-18 September 2014.

8. Barai, A.; Chouchelamane, G.H.; Guo, Y.; McGordon, A.; Jennings, P. A study on the impact of lithium-ion cell relaxation on electrochemical impedance spectroscopy. J. Power Sources 2015, 280, 74-80. [CrossRef]

9. Wang, X.; Wei, X.; Dai, H. Estimation of state of health of lithium-ion batteries based on charge transfer resistance considering different temperature and state of charge. J. Energy Storage 2019, 21, 618-631. [CrossRef]

10. Guha, A.; Patra, A. Models, tate of health estimation of lithium-ion batteries using capacity fade and internal resistance growth. IEEE Trans. Transp. Electrif. 2017, 4, 135-146. [CrossRef]

11. Gismero, A.; Stroe, D.-I.; Schaltz, E. Calendar aging lifetime model for NMC-based lithium-ion batteries based on EIS measurements. In Proceedings of the 2019 Fourteenth International Conference on Ecological Vehicles and Renewable Energies (EVER), Monte-Carlo, Monaco, 8-10 May 2019.

12. Xiong, R.; Tian, J.; Mu, H.; Wang, C. A systematic model-based degradation behavior recognition and health monitoring method for lithium-ion batteries. Appl. Energy 2017, 207, 372-383. [CrossRef]

13. Carthy, K.M.; Gullapalli, H.; Ryan, K.M.; Kennedy, T. Use of Impedance Spectroscopy for the Estimation of Li-ion Battery State of Charge, State of Health and Internal Temperature. J. Electrochem. Soc. 2021, 168, 080517. [CrossRef]

14. Plett, G. Extended Kalman filtering for battery management systems of LiPB-based HEV battery packs Part 3. State and parameter estimation. J. Power Sources 2004, 134, 277-292. [CrossRef]

15. Urbain, M.; Rael, S.; Davat, B.; Desprez, P. State estimation of a lithium-ion battery through kalman filter. In Proceedings of the 2007 IEEE Power Electronics Specialists Conference, Orlando, FL, USA, 17-21 June 2007.

16. Remmlinger, J.; Buchholz, M.S.-G.T.; Dietmayer, K. On-board state-of-health monitoring of lithium-ion batteriesusing linear parameter-varying models. J. Power Sources 2013, 239, 689-695. [CrossRef]

17. Vetter, J.; Novak, P.; Wagner, M.R.; Veit, C.; Möller, K.-C.; Besenhard, J.; Winter, M.; Wohlfahrt-Mehrens, M.; Vogler, C.; Hammouche, A. Ageing mechanisms in lithium-ion batteries. J. Power Sources 2005, 147, 269-281. [CrossRef]

18. Westerhoff, U.; Kurbach, K.; Lienesch, F.; Kurrat, M. Analysis of Lithium-Ion Battery Models based on Electrochemical Impedance Spectroscopy. Energy Technol. 2016, 4, 1620-1630. [CrossRef]

19. Tian, L.; Zhuang, Q.; Li, J.; Shi, Y.; Chen, J.; Lu, F.; Sun, S. Mechanism of intercalation and deintercalation of lithium ions in graphene nanosheets. Chin. Sci. Bull. 2011, 56, 3204-3212. [CrossRef]

20. Kallel, A.Y.; Bouchaala, D.; Kanoun, O. Critical implementation issues of excitation signals for embedded wearable bioimpedance spectroscopy systems with limited resources. Meas. Sci. Technol. 2021, 32, 084011. [CrossRef] 